\title{
Reactions of Persons with Dementia to Caregivers Singing in Morning Care Situations
}

\author{
Lena Marmstål Hammar ${ }^{* 1,2}$, Azita Emami ${ }^{1,3}$, Gabriella Engström² ${ }^{2}$ and Eva Götell ${ }^{1,2}$ \\ ${ }^{I}$ Department of Neurobiology, Care Science and Society, Division of Nursing, Karolinska Institute, Sweden \\ ${ }^{2}$ School of Health, Care and Social Welfare, Mälardalen University, Sweden \\ ${ }^{3}$ College of Nursing, University of Seattle, USA
}

\begin{abstract}
Music therapeutic caregiving', when caregivers sing for or together with persons with severe dementia during care situations, has been suggested as a way to reduce problematic behaviors in dementia care. The present study implemented this technique as an intervention in dementia care. Six caregivers participated in group interviews about their experiences of morning care situations without and with'Music therapeutic caregiving'. Through a qualitative content analysis two themes emerged.'Being in a different reality' was based on'usual' morning care situations. The caregivers' experienced the persons with dementia as absent-minded; communication and cooperation were difficult. The second theme,'Being present', was based on morning care situations with the intervention. The caregivers described communication as enhanced; the persons with dementia expressed themselves more appropriately, making cooperation possible. The results indicate that 'Music therapeutic caregiving' might lead to a more positive experience of the person with dementia and seems to increase receptivity to caregiving.
\end{abstract}

Keywords: Dementia, caregiver, singing, intervention, experience, qualitative content analysis.

\section{INTRODUCTION}

It is well-known that persons with dementia (PWDs) react with so-called behavioral and psychological symptoms of dementia (BPSD), a range of behaviors such as verbal and physical aggression, resisting care, wandering, and screaming [1]. BPSD make caring for PWDs problematic and earlier research argues that such behavior can neither be hindered nor resolved. When dealing with this problematic situation caregivers might resort to constraining PWDs, which commonly exacerbates agitated behavior [2, 3]. Research suggests that PWDs' aggressive behaviors are rooted in communication difficulties. These individuals have an impaired ability to interpret and express non-verbal and verbal communication, and linguistic functions are commonly affected, leading them to communicate with aggression because words are unavailable [4]. Thus, improving communication is crucial and several strategies have been investigated in an attempt to facilitate communication and minimize BPSD [5].

In order to make life as meaningful as possible for PWDs and their caregivers, and to address the challenges that can arise in relation to behaviors PWDs sometimes exhibit, earlier research suggests that non-pharmacological treatment, such as caring interventions, should be applied [6-9]. Such treatment could include music therapy or use of music in dementia care $[10,11]$. Furthermore, the Swedish Council on Technology Assessment in Health Care [12] found that it is possible to communicate and establish positive relationships with PWDs and bring out their latent abilities when using

*Address correspondence to this author at the Mälardalen University, P.O. Box 883, SE 72123 Västerås, Sweden; Tel: +70 60205 33; Fax: +46 2110 16 33; E-mail: lena.marmstal.hammar@mdh.se music, dancing, and singing. In a larger project using qualitative methods, Götell [13] analyzed video-recorded observations, focusing on communication between persons with severe dementia and professional caregivers during morning care situations. First, a morning care situation was carried out the'usual' way. Thereafter an intervention was made that involved the caregivers singing for or together with the PWDs, so-called'Music Therapeutic Caregiving' (MTC) [14]. It was clearly advantageous to use MTC, and the authors were surprised to discover that PWDs' aggressive behavior and screaming were abated, while verbal communication between caregivers and PWDs was enhanced [15]. PWDs demonstrated better posture, movements and sensory awareness [16], and responded with more vitality and expressed positive emotions and moods [17]. However, the caregivers' experiences of using MTC were not explored. The first study to investigate this issue was conducted to describe professional caregivers' experiences of caring for persons with dementia during morning care situations, focusing on the caregivers' own feelings and actions both without and with MTC. Results revealed that they discovered "the key to communion" with the PWDs through the use of MTC. With MTC the caregivers were able to communicate with the PWDs in a pleasant manner and they experienced a sense of well- being because they no longer had to constrain the PWDs in care situations [18]. The present study describes professional caregivers' experiences of the PWDs in the context of morning care situations with and without MTC, as this has not earlier been investigated.

\section{AIM}

The aim was to present professional caregivers' experiences of persons with dementia during morning care situations without and with Music Therapeutic Caregiving. 


\section{METHOD}

This paper reports on the results of a study conducted within the context of a larger research project involving a dementia care intervention [19]. The overall aim of the larger research project was to implement MTC in morning care situations and evaluate the influences this might have upon PWDs and their professional caregivers. The research project was conducted at two nursing homes for PWDs in an urban area of Sweden and the study participants included PWDs and their caregivers. At our request, the head nurses at each of these homes suggested ten female caregivers for participation in our study. Two women declined due to health issues, one withdrew her participation, and one terminated her participation since the PWD she was caring for passed away. Thus, three caregivers from each home participated in the research project, four of whom were trained as assistant nurses and two were nurse aides. Participants were between 31-54 years old, had been working in dementia care between 2.5 and 30 years, and were native Swedish speakers. All of the caregivers had extensive experience of caring for the PWDs participating in the project. The head nurses also suggested the ten PWDs who participated in the study. Of these ten PWDs, six were women and four were men. According to their medical records, five of the PWDs were diagnosed with Alzheimer's disease and five were diagnosed with vascular dementia. To elucidate cognitive function in the PWDs, Mini-mental state examinations (MMSE) [20] were conducted by the first author. The resulting scores were between 12-0, and the mean value was 3.3, which indicated that all the PWDs had severe dementia. The study presented here involved the caregivers who participated in the research project (described above) with an aim to investigate their experiences of the PWDs participating in the research project (described above), during morning care situations without and with Music Therapeutic Caregiving.

The research situation was morning care situations, which consisted of the caregiver helping the PWD from the bed to the bathroom. The caregiver removed the PWD's nightclothes, while the PWD remained seated on the toilet. The PWD's face and upper body were washed and lotion and deodorant were applied. The PWD's abdomen was bathed. Clothes, socks and shoes were put on, and the PWD stood up and was led to the sink (three of them in wheelchairs) and directed towards the mirror. While at the sink, the PWD's teeth were brushed (by the caregiver or by the PWD), and their hair was combed (by the caregiver or by the PWD). The morning care situation ended when the PWD and the caregiver left the bathroom. The'usual' morning care situation (as described above) provided a baseline [19] against which we could study the effects of an intervention [19]. The intervention was comprised of morning care situations that followed the same steps as in'usual' morning care situations but with MTC - the caregivers sang for or together with the PWDs while caring for them. That is, the'usual' morning care situation was compared with the'intervention situation'. The'usual' morning care situations were exercised by the participants once a week over a 4-week period and then the intervention situation was upheld for an equal amount of time. The same caregiver-PWD pairs (in all 10 pairs) participated in the'usual' morning care situations and in the intervention situations.
To prepare the caregivers for the MTC-intervention they were offered a course in MTC at Mälardalen University, which included theories about MTC [13], music and health, using the singing voice, body movements to accompany singing, learning songs from elderly people's childhood and young adult years, and how to communicate with PWDs through singing. Two of the participants took the course, while the others were instructed on how to use MTC by the first author, who was certified in MTC. During the intervention, songs were sung that were easy to recognize for older people, including sing-along songs, children's songs and popular songs from the early part of the $20^{\text {th }}$ century. The caregivers themselves chose which songs they preferred to sing and they sang continuously. Some of them spoke between the songs, but only to instruct or explain something to the PWD. Since they sang more or less continuously, they sang as long as the morning care situations took place, between 10 to 20 minutes.

\section{Ethical Considerations}

The Regional Board of Research Ethics approved the study. All caregivers were informed orally and in writing about the study and research ethics by the first author. The caregivers then signed a written consent. The PWDs in the study were fragile persons, and because of their severe dementia they were unable to understand the information given about the study. Therefore, proxy consent [21] was obtained from the PWDs' next of kin. Additionally, the researcher carefully observed the PWDs for any signs that indicated that they objected to participating in the study or that their integrity was being compromised. No such signs were observed.

\section{Data Collection}

In order to capture the caregivers' descriptions of their experiences from morning care situations, a qualitative approach with qualitative interviews was suitable. Group Interviews [22] were conducted with the caregivers, and the first author (LM) carried these out as a moderator with assistance from the last author (EG). The interviews were recorded and lasted about one hour each, and were unstructured with open-ended questions for discussion in the groups under the moderator's guidance. One Group Interview was conducted at each home before the intervention and one at each home following the intervention, in all four interviews.

The Group Interviews that were conducted after'usual' morning care situations began with one specific question,' Could you please tell me about your experiences in caring for the PWDs during morning care situations?' This question was followed-up with probing questions [19]. The question asked in Group Interviews following the intervention was,'Could you please tell me about your experiences in caring for the PWDs during morning care situations with MTC?' Also this question was followed-up with probing questions.

\section{Data Analysis}

The interviews were transcribed verbatim; including repetition, laughter, silence, etc. A latent qualitative content analysis [23] was conducted to analyze the resulting texts. All four interviews were intially analyzed as one text, and 
the first author read the text several times to get a sense of it in its entirety. At this point two content areas emerged,'being in a foreign situation', which was based on the interviews about the'usual' morning care situations, and'to recognize the situation', which was based on the interviews about the MTC situations. Henceforth, texts related to the interviews about the'usual' morning care situation and the interviews about the MTC situation were analyzed separately. Next, the text was extracted into meaning units, which consisted of words, sentences or phrases related to each other through their content and the context. The meaning units were condensed, and labeled with codes, which referred to discrete events, objects or other phenomena that can be understood in relation to the context. Codes were continuously compared to identify differences and similarities, and based on the codes, categories were developed; these categories were red threads that appeared throughout the codes. The meaning units, codes and categories dealt with the manifest content of the text. The next step was to compare and critically analyze the categories on a more abstract and interpretive level, and eight sub-themes were developed. Four sub-themes were based on the interviews about the'usual' morning care routines, and four were based on the interviews about the MTC situations. A theme links the underlying meanings in the categories together, and is a red thread throughout the condensed meaning units, codes and categories on an interpretative level, and expresses the latent content of the text. The eight sub-themes formed two comprehensive themes, i.e. Being in a different reality, which was based on interviews about the'usual' morning care situations and Being present, which was based on the interviews about the MTC situations.

\section{RESULT}

\section{Being in a Different Reality}

According to the caregivers' descriptions, it was difficult to connect with the PWDs in the morning care situation. PWDs were sometimes described as resistant and aggressive, making communication and cooperation difficult. Participants stated that the PWDs occasionally expressed affection, but they still seemed to be aloof to the situation.

\section{Being Aloof}

According to the caregivers, communication difficulties were a significant obstruction, and the PWDs were commonly said to be physically but not mentally present in the care situation. Their responses to questions or requests were confused, if they responded at all, when caregivers spoke to them, and they often did not make eye contact. Caregivers described some of the PWDs' eyes as'staring out into empty space'. One caregiver expressed her experiences as such:

'He is in his own little world, and he mostly seems confused and surprised when I speak to him'.

The PWDs commonly lacked the ability to interpret verbal and non-verbal communication, as well as the ability to express it. Instead, the PWDs' communication often involved behaviors such as resistance and sometimes aggression as well as expressions of insecurity and anxiety, which made it difficult to care for them.

\section{Showing Resistance}

Caregivers reported that resistance arose when the PWDs did not want to, or were unable to, cooperate. Resistance could take place at all stages of the morning care situation, but mostly while undressing, washing, or giving the PWDs instructions. Some PWDs responded with irritation and verbal protests, or by physically showing their resistance by moving reluctantly. The caregivers stated that resistance surfaced because the PWDs were afraid, or were unable to understand the situation and the demands being made of them. According to one caregiver:

'Sometimes I think they do not mean to be resistant, but it's as if their brains cannot keep it together and know what to do'.

Resistance was described as being common while brushing teeth. Sometimes, the PWDs did not want to open their mouths and tried to avoid the activity, which in turn could have a negative impact on their oral hygiene.

\section{Showing Aggression}

Some PWDs did express physical and verbal aggression. Some were aggressive while getting undressed and some during washing or both. Aggression commonly escalated while the PWDs were being washed, and might involve the PWDs throwing things, slapping, cursing, screaming or issuing insults; their behavior was described as unpredictable, and it sometimes resulted in scratches and bruises on the caregivers. One caregiver expressed her experience of aggression as such:

'It is like they get so stressed out, and they
cannot express it in any way other than being
aggressive... and once they have started to
fight it is almost impossible to get them to
stop'.

Aggression was believed to surface because the PWDs did not understand the situation, and due to communication difficulties. The PWDs seemed scared or confused and were unable to express this other than through aggression.

\section{Expressing Affection}

The caregivers suggested that the PWDs sometimes expressed affection in or after the'usual' morning care situation. One caregiver stated:

'Even though she does not realize who I am, she expresses nice things now and again'.

The PWDs sometimes gave compliments or gently stroked the caregivers.

\section{Being Present}

The caregivers' described their experiences with the PWDs differently following MTC; communication was enhanced and PWDs could more often express themselves more adequately, making cooperation possible. The PWDs were described as generally more compliant and relaxed, and they expressed joy by singing and laughing. 


\section{Being Engaged and Empowered}

The caregivers described MTC as a possible way to engage with the PWDs, and pointed out that the PWDs were more cooperative, without the caregiver needing to make demands or give instructions about what to do. One caregiver described communication in the following way:

'It is strange, because it feels like she also communicates.... just like she wants to participate, and I.... I do not talk that much. It is strange when I think about it....'.

The PWDs were described as present and focused on the situation, and they seemed interested in what was going on. They watched the caregiver with curiosity. As one caregiver put it:

'Before it was only me focusing on the situation, now it is both.... It is mutual in some way'.

The caregivers described eye contact with the PWDs, which was regarded as a fundamental component in communication. Some PWDs initiated communication, and the caregivers described the PWDs as more cohesive, and communication as more'face-to-face'.

The PWDs requested instructions less frequently during MTC, and when speaking to the caregivers the PWDs commonly responded more appropriately. Some PWDs spoke more and some answered with sentences rather than with single words. The PWDs' speech flowed more smoothly, and some commented on how the caring should be carried out. As a conclusion in the interview about MTC, one caregiver described it by saying:

'The coherent thread is that they become present and alive'.

The PWDs were described as peaceful and secure, and as making pleasant comments, as for example in this caregiver's recollection:

\section{'She looked into my eyes and said that they were beautiful'.}

One out of ten PWDs was described as aggressive when morning care routines began, while being undressed and while being washed. The caregiver stated that the PWD was in need of verbal instructions, and mixing songs with verbal instructions enhanced communication. While getting dressed, the PWD enjoyed singing along, and cooperated and communicated in an engaged way.

\section{Demonstrating Episodes of Lucidity}

Some PWDs sang along during MTC, and some PWDs even corrected the caregivers when they sang texts, and some ended the song if the caregivers stopped singing or forgot the lines of a song. One PWD made up her own song text to jest with the caregiver. Some PWDs commented on the song texts as if they were in a conversation with the song that the caregivers sang. The caregivers also described how some PWDs requested songs they wanted to hear. Some PWDs replied correctly to the caregivers' requests and spontaneously started to speak or sing, as described by one caregiver: '...she started to speak sentences, which she had not done in a long time'.

The caregivers described the PWDs as expressing lucidity through cooperating in the care situation. Some of them began to wash themselves or tried to undress or dress themselves without being told to do so. The caregivers regarded the singing as the source of the PWDs' understanding of what to do, as stated in this example:

\section{'She took the washcloth and washed her hands without me telling her to do so. I had not seen that before'.}

The caregivers also reported that some PWDs noticed themselves in the mirror. One PWD combed her own hair when the caregiver already had done so, because she wanted it another way. Another PWD noticed herself in the mirror and made jokes about her own reflection.

\section{Becoming "Compliant"}

Although some PWDs did not cooperate during MTC, they did not attempt to avoid caregiving tasks or become resistant. The caregivers described them as being "compliant"; they accepted the situation. As an example, caregivers stated that they did not have to "bend the PWDs' arms to get them dressed or care for them. According to one caregiver:

'She did not try to escape as much.... as if she didn't mind me taking care of her'.

Moreover, MTC was described as also making it easier to brush teeth because the PWDs let the caregivers do so without struggling to avoid it. The caregivers noted that the PWDs moved with strength and self-confidence during MTC, and they more easily stood up, walked, and showed purpose in their movements. The pace of the songs was described as helping them walk easier, with strength in their steps.

\section{Expressing Joy and Relaxation}

According to the caregivers' reports, the PWDs looked happy and sometimes even laughed and made jokes. Some made faces and hummed along when the caregiver sang, and some PWDs said that they thought it was fun when the caregivers sang, and when the caregivers sang the wrong text some PWDs laughed. One caregiver commented:

'If we sing the wrong text or if she thinks it sounds funny, she laughs at us and rolls her eyes'.

One PWD was described as enjoying the singing so much that she forgot what she was supposed to do, which made the care situation last longer, but it was nevertheless more enjoyable.

The singing was regarded as bringing together the different elements of the care situation and making it run smoothly, in a relaxed manner. The PWDs' faces and bodies were described as relaxed, and some of the PWDs leaned back in a casual manner and let the caregiver take care of them. In the words of one caregiver:

\section{'It is like she has let out a deep, pleasant sigh'.}


Some PWDs were alert and wanted to cooperate in the care situation, in a relaxed and self-confident way, and they were described as being relaxed but active. One PWD was described as aggressive at the start of the morning care situation, when she was being undressed and washed, but were described to be relaxed and happy by the time she was being dressed.

\section{DISCUSSION}

\section{Methodological Considerations}

Data for the study were collected through Group Interviews. This data collection method is considered suitable when the researcher wants to gather a group's experiences of a certain subject [22]. The Group Interviews conducted for this study led to open discussions between the participants. Through conversation, agreements and disagreements emerged that enriched the interviews. One possible disadvantage of using Group Interviews is that the participants and the first author were familiar with one another and this could have influenced the honesty of responses. However, our impressions were that the Group Interviews were characterized by openness and lively descriptions of both positive and negative situations.

The caregivers were somewhat familiar with outcomes that had earlier been achieved with MTC [13] and two of them had taken a course in MTC (Music Therapeutic Caregiving I, MKM019), which might have made them consciously look for an effect while they were singing. Similarly, the first author, who performed the analysis, might have been biased by knowledge of previous research [13, 18]. The interpretation of the text should therefore be seen as one possible interpretation of the caregivers' descriptions of their experiences of the situations.

Qualitative content analysis [23] was useful for structuring the text and allowed us to move back and forth between the whole and parts of the material. Because of the clear differences between the descriptions of the'usual' morning care situation and the MTC intervention situation this was critical as a means of increasing trustworthiness. Trustworthiness was also increased by performing the analysis in cooperation with the researchers in this paper (LM, EG, AE, GE). Furthermore, the manuscript was critically discussed in a scientific group, and the transcribed text of the interviews, as well as the findings, were presented to the caregivers who confirmed the findings.

\section{Reflections on the Results}

The study aimed to present professional caregivers' experiences of PWDs during morning care situations without and with MTC. Two different descriptions emerged. In relation to the'usual' morning care situation, the caregivers described problematic behaviors such as aggression and resistance that were exhibited by the PWDs. These statements gave rise to the first theme -'Being in a different reality'. According to this theme, PWDs were described as being absent from the situation and communication and cooperation were difficult or even impossible. The PWDs sometimes expressed affection, but remained aloof in the situation. When describing the morning care situation with MTC, the caregivers pointed out that they experienced enhanced communication and more appropriate responses from the PWDs, making cooperation possible. These descriptions formed the second theme -'Being present'. The PWDs were present in the situation and communicated with enhanced vitality, expressed joy, sang along, laughed, and were compliant.

In relation to the first theme,'Being in a different reality', the caregivers described the PWDs as not being mentally present in the situation, which could be related to what Finkel [1] has called apathy or psychotic behavior, and which is included in the concept BPSD. This study also revealed that the caregivers experienced the PWDs as having difficulties interpreting and expressing communication, and as using aggression and resistance to express themselves. Similar results were found by Acton, Yauk, Hopkins and Mayhew [4], who suggested that the PWDs' aggressive behavior surfaces because of difficulties in communication. In this study, the research situations were morning care situations. The caregivers and the PWDs interacted very closely with one another in ways that could involve the caregivers intruding on the PWDs' personal space. As Brodaty and Low [24] suggest, aggressive behavior is a response from the PWD when someone intrudes upon their personal space. This study further revealed that the caregivers believed that the PWDs' aggression and resistance surfaced because they did not understand the situation or were unable to communicate with the caregiver. McDaniel, Edland and Heyman [25] suggest that when PWDs encounter problems in relating to themselves, to others, and to the world, situations in which they interact with others become problematic. The interaction between the PWDs and the caregivers is crucial, however, and according to this study, communication and cooperation seemed hindered, because the participants operated in "different realities".

The second theme,'Being present', which was based on the MTC situations, encompassed very different descriptions, and this is the first study that we know of that is based on caregivers' experiences of MTC, focusing on the PWDs. Several studies have examined the use of music in dementia care, but most are based on situations outside caring activities, such as music therapy [26]. Other studies have focused on playing background music or listening to music in care situations [27-29]. MTC is unique because it is implemented as an intervention in a care situation in which the PWDs and the caregivers interact to receive and carry out care, respectively. This study revealed that when MTC was introduced, the PWDs were described as present and focused on the morning care situation, and this confirms findings from previous studies about MTC by Götell [13]. The PWDs' focus on the situation made communication between them and the caregivers possible, which was also shown by Götell, Brown and Ekman [15]. In this study, the caregivers, based on their experiences, stated that verbal communication was not as necessary during MTC, and that they did not instruct the PWDs as much in what to do, but the PWDs were compliant and able to cooperate nonetheless. Similar results were found by Götell, Brown and Ekman [16, 17]. Results of this study further revealed that the PWDs were described as present, and inviting, and seemed to be willing to interact with the caregiver. In turn, they were able to work together to a greater extent, and it was easier to carry out care work. Similar results were found by Götell, Brown and Ekman [30]. Ekman [31] and Normann, Asplund, Karlsson, 
Sandman and Norberg [32] as well as Normann, Asplund and Norberg [33] suggest that too much verbal communication and too many instructions might be challenging for the PWD to handle. Avoiding demands and seeing the PWD as a whole human being resembles'communion' in the encounter. One interpretation of the caregivers' experiences of the PWDs when using MTC is that the two parties were working in communion, and as such, were able to communicate and cooperate. In addition, the PWDs were described as communicating verbally and non-verbally more successfully; they remembered song texts, and were able to sing along, and could even instruct the caregivers about how they wanted things to be done. Ridder and Aldridge [26], suggest that PWDs are able to remember all the words to a song but rarely speak or are able to put sentences together. These incidences could be understood as'episodes of lucidity', which are possible when communion between caregivers and PWDs is reached [32, 33]. We were surprised that the caregivers described such a strong influence on the PWDs when they sang. Additionally, O'Callaghan [34] and Stewart, von Kriegstein, Warren and Griffiths [35] can shed light on this, as they suggest that music allows access to different parts of the brain, based on the many elements involved in music, such as rhythm, melody, timbre, accent, etc. Language is a function of the left hemisphere, and a combination of language and music, as in singing, presents a greater chance of activating intact neurological pathways than using language alone.

\section{CONCLUSION}

In care situations, such as the morning care situations studied here, the aim is to perform or receive care. According to the accounts given by the caregivers who experienced a change during the situations in this study, and in Hammar Marmstål, Emami, Engström and Götell [18], it is of great importance to be able to interact well with the PWD to make the care situation as pleasant as possible, and the care work easier to perform. MTC seemed to instigate a positive encounter, which contributed to a relaxed care situation that ran smoothly. Paterson and Zderad [36] note the importance of the relationship in the care situation, and suggest that the interaction between the PWD and the caregiver is characterized by'being' and'doing': Care interventions should not only consist of providing care 'doing' - but should also involve'being' with the patient because this has a positive influence on the care situation. Based on the results of this study and previous research by Götell, Brown and Ekman [15-17], as well as Hammar Marmstål, Emami, Engström and Götell [18], we suggest that MTC could be an effective non-pharmacological nursing treatment that offers the possibility for PWDs and the caregivers to interact positively in care situations because it facilitates them'being' together in the situation. Based on the results descriptions, we also suggest that MTC is a possible method to help PWDs express their latent abilities and thereby communicate as whole human beings with their caregivers during morning care situations. However, we are aware that more research is needed and, as suggested by Kverno, Black, Nolan, and Rabins [9], intervention studies carried out in the context of personal care for persons with advanced dementia are lacking. Our own study contributes to knowledge in this area. The next step in this project will be to analyze video-observations of the morning care situations with and without MTC to further explore what influences MTC might have on PWDs and the relationship between them and their caregivers.

\section{ACKNOWLEDGEMENTS}

We wish to thank the caregivers that participated in this study. Thanks also to Ms. Caroline Sutton for the linguistic revision. The study is financed by a grant from Sparbanksstiftelsen Nya, Johanniterorden i Sverige, Västerås Stad, Demensförbundet, and Mälardalen University and supported by the College of Nursing at Seattle University, USA, and Department of Neurobiology, Care Science, and Society, Division of Nursing, Karolinska Institute, Sweden.

\section{CONFLICT OF INTEREST AND FUNDING}

The authors of the study have received no benefits from industries that would imply a conflict of interest in this study.

\section{CONTRIBUTORS}

Study design: LM, AE, GE, EG, data collection: LM, $\mathrm{EG}$, data analysis: $\mathrm{LM}, \mathrm{AE}, \mathrm{GE}, \mathrm{EG}$, and manuscript preparation: LM, AE, GE, EG.

\section{ABBREVIATIONS}

$$
\begin{aligned}
\text { PWDs }= & \text { Persons with dementia } \\
\text { BPSD }= & \text { Behavioral and Psychological Symptoms of } \\
& \text { Dementia } \\
\text { MTC }= & \text { Music Therapeutic Caregiving } \\
\text { MMSE }= & \text { Mini Mental State Examination }
\end{aligned}
$$

\section{RERERENCES}

[1] Finkel SI. Behavioral and psychologic symptoms of dementia. Clin Geriatr Med 2003; 19(4): 799-824.

[2] Graneheim UH, Isaksson U, Ljung IM, Jansson L. Balancing between contradictions: the meaning of interaction with people suffering from dementia and "behavioral disturbances". Int J Aging Hum Dev 2005; 60(2): 145-57.

[3] Skovdahl K, Kihlgren AL, Kihlgren M. Different attitudes when handling aggressive behaviour in dementia -- narratives from two caregiver groups. Aging Ment Health 2003; 7(4): 277-86.

[4] Acton GJ, Yauk S, Hopkins BA, Mayhew PA. Increasing social communication in persons with dementia. Res Theory Nurs Pract 2007; 21(1): 32-44.

[5] Small JA, Gutman G, Makela S, Hillhouse B. Effectiveness of communication strategies used by caregivers of persons with Alzheimer's disease during activities of daily living. JSLHR: J Speech Lang Hear Res 2003; 46(2): 353-67.

[6] Agency MP. In Engelish: Drug treatments and encounter of BPSD in'demantia care. In Swedish: Läkemedels behandling och bemötande vid beteendemässiga och Psykiska Symtom vid demenssjukdom - BPSD. Information from The Medical Products Agency, Sweden 2008.

[7] Cohen-Mansfield J, Marx MS, Thein K, Dakheel-Ali M. The impact of past and present preferences on stimulus engagement in nursing home residents with dementia. Aging Ment Health 2010; 14(1): 67-73.

[8] Herrmann N, Gauthier S. Diagnosis and treatment of dementia: 6. Management of severe Alzheimer disease. Can Med Assoc J 2008; 179(12): 1279-87.

[9] Kverno KS, Black BS, Nolan MT, Rabins PV. Research on treating neuropsychiatric symptoms of advanced dementia with nonpharmacological strategies, 1998-2008: a systematic literature review. Int Psychogeriatr 2009; 21(5): 825-43. 
[10] Lelchuk Staricoff R. Arts in health: a review of the medical literature 2004. Research report36ArtCouncilEnglandhttp://wwwa rts councilorguk/documents/publications/php7FMawEdoc

[11] Skingley A, Vella-Burrows T. Therapeutic effects of music and singing for older people. Nurs Stand 2010; 24(19): 35-41.

[12] The Swedish Council on Technology Assessment in Health Care S. Dementia - Caring, Ethics, Ethnical and Economical Aspects- A Systematic Review. Mölnlycke: Elanders Infologistics Väst AB 2008.

[13] Götell E. Singing, background music and music-events in the communication between persons with dementia and their caregivers. 2003 [cited; 82 s.]. Available from: http: //diss.kib.ki.se/2003/91-7349-613-8/

[14] Brown SG, Götell E, Ekman S-L. Music-therapeutic caregiving': the necessity of active music-making in clinical care. Arts Psychother 2001; 28 125-35

[15] Götell E, Brown S, Ekman S. Caregiver singing and background music in dementia care. West J Nurs Res 2002; 24(2): 195-216.

[16] Götell E, Brown S, Ekman S. Influence of caregiver singing and background music on posture, movement, and sensory awareness in dementia care. Int Psychogeriatr 2003; 15(4): 411-30.

[17] Götell E, Brown S, Ekman SL. The influence of caregiver singing and background music on vocally expressed emotions and moods in dementia care: a qualitative analysis. Int J Nurs Stud 2009; 46(4): 422-30.

[18] Hammar Marmstål L, Emami A, Engstöm G, Götell E. Finding the key to communion - Caregivers experience of music therapeutic caregiving in dementia care. A qualitative analysis. Dementia The International Journal of Social Research and Practice 2011; in press.

[19] Polit DF, Beck CT. Nursing research: generating and assessing evidence for nursing practice. $8^{\text {th }}$ ed. Philadelphia: Wolters Kluwer Health/Lippincott Williams \& Wilkins 2008.

[20] Folstein MF, Folstein SE, McHugh PR. "Mini-mental state". A practical method for grading the cognitive state of patients for the clinician. J Psychiatr Res 1975; 12(3): 189-98.

[21] Karlawish J, Kim SY, Knopman D, van Dyck CH, James BD, Marson D. The views of Alzheimer disease patients and their study partners on proxy consent for clinical trial enrollment. Am J Geriatr Psychiatry 2008; 16(3): 240-7.

[22] Côte Arsenault D, Morrison Beedy D. Focus on research methods. Maintaining your focus in focus groups: avoiding common mistakes. Arthritis Care Res 2005; 28(2): 172-9.
[23] Graneheim UH, Lundman B. Qualitative content analysis in nursing research: concepts, procedures and measures to achieve trustworthiness. Nurse Educ Today 2004; 24(2): 105-12.

[24] Brodaty H, Low LF. Aggression in the elderly. J Clin Psychiatry 2003; 64: 36-43.

[25] McDaniel KD, Edland SD, Heyman A. Relationship between level of insight and severity of dementia in Alzheimer disease. CERAD Clinical Investigators. Consortium to Establish a Registry for Alzheimer's Disease. Alzheimer Dis Assoc Disord 1995; 9(2): 1014.

[26] Ridder HM, Aldridge D. Individual music therapy with persons with frontotemporal dementia: singing dialogue. Nord J Music Ther 2005; 14(2): 91-106.

[27] Gerdner LA. Effects of individualized versus classical "relaxation" music on the frequency of agitation in elderly persons with Alzheimer's disease and related disorders. Int Psychogeriatr 2000; 12(1): 49-65

[28] Ragneskog H, Brane G, Karlsson I, Kihlgren M. Influence of dinner music on food intake and symptoms common in dementia. Scand J Caring Sci 1996; 10(1): 11-7.

[29] Ragneskog H, Kihlgren M. Music and other strategies to improve the care of agitated patients with dementia: interviews with experienced staff. Scand J Caring Sci 1997; 11(3): 176-82.

[30] Götell E, Brown S, Ekman SL. Caregiver-assisted music events in psychogeriatric care. J Psychiatr Ment Health Nurs 2000; 7(2): 119.

[31] Ekman SL. Monolingual and bilingual communication between patients with dementia diseases and their caregivers. Int Psychogeriatr 1996; 8(Suppl 1): 127-32.

[32] Normann HK, Asplund K, Karlsson S, Sandman P, Norberg A. People with severe dementia exhibit episodes of lucidity. A population-based study. J Clin Nurs 2006; 15(11): 1413-7.

[33] Normann HK, Asplund K, Norberg A. Episodes of lucidity in people with severe dementia as narrated by formal carers. J Adv Nurs 1998; 28(6): 1295-300.

[34] O'Callaghan CC. Communicating with brain-impaired palliative care patients through music therapy. J Palliat Care 1993; 9(4): 53-5.

[35] Stewart L, von Kriegstein K, Warren JD, Griffiths TD. Music and the brain: disorders of musical listening. Brain 2006; 129(Part 10): 2533-53.

[36] Paterson J, Zderad L. Humanistic Nursing. New York: National league for nursing 1988 .

(C) Hammar et al.; Licensee Bentham Open.

This is an open access article licensed under the terms of the Creative Commons Attribution Non-Commercial License (http://creativecommons.org/licenses/by$\mathrm{nc} / 3.0 /$ ) which permits unrestricted, non-commercial use, distribution and reproduction in any medium, provided the work is properly cited. 